\title{
Assessment of gaseous phase PAHs emitted from marine diesel engine using vortex-assisted dispersive liquid-liquid microextraction followed by GC/MS
}

\author{
M.Y. WANG ${ }^{1}$, S.J. Zhang ${ }^{2}$, C.J. Wang ${ }^{2}$ \\ ${ }^{1}$ Ocean School, Yantai University, 32 Qingquan Road, 264005 Yantai, China \\ ${ }^{2}$ Maritime College, Shandong Jiaotong University, 115 Xinwei Road, 264200, Weihai, China
}

Keywords: PAH; Emission; Detection; Vortex-assisted dispersive liquid-liquid microextraction

\begin{abstract}
A novel sample treatment, namely vortex-assisted dispersive liquid-liquid microextraction (VDLLME), followed by GC/MS has been developed for the determination of gaseous phase polycyclic aromatic hydrocarbons (PAHs) emitted from a marine diesel engine. The method integrates extraction, enrichment, and concentration into one step by combining sample collection and VDLLME into a much shorter and accurate procedure. We have optimized several important variables influencing the extraction efficiency including type and amount of extraction solvent, type and volume of disperser solvent, sample $\mathrm{pH}$, salting-out effect, vortex and centrifugation time. Under the optimum conditions, high enrichment factors (more than 1,000) and acceptable total spiked recoveries (72.0-95.1\%) were obtained using GC/MS detection. The limits of detection and quantification of the proposed method were in the ranges of 0.1-0.5 ng/L. Our total pretreatment can be competed in less than 0.5 hours so as to decreases loss of PAHs by $18.37 \%$ compared to traditional methods.
\end{abstract}

\section{Instruction}

Marine diesel engines are significant contributors to air pollution in many coastal areas. Their exhaust emissions have been found to contain numerous toxic air contaminants including the highly carcinogenic polycyclic aromatic hydrocarbons (PAHs). PAHs, compounds created during the process of incomplete combustion of the organic matter, not only have carcinogenic, teratogenic and mutagenic effects, but also can lead to the formation of other highly toxic substance (nitroPAHs, hydroxy-PAHs) through reactions with other pollutants such as ozone, hydroxyl radicals, nitrogen, and sulfur oxides (Khamphe et al. 2013). Therefore, accurate collection and quick analysis of PAHs emissions of diesel engines is crucial to better assess health risks in coastal areas.

Rezaee suggested the dispersive liquid-liquid microextraction (DLLME), which is based on the ternary component solvent system (Rezaee et al. 2006). DLLME is simple to operate, and is an especially rapid and inexpensive extraction method with high preconcentration factors. This technique has successfully been applied to preconcentration of PAHs, drugs and pesticides (Natalia et al. 2015; Fan et al. 2015). To enhance dispersion of hydrophobic ionic liquids (extraction solvent) into the aqueous sample, vortex was introduced, this methodology, named vortex-assisted DLLME (VDLLME) offers the advantage of avoiding the possible degradation of some analytes. VDLLME has been applied for the analysis of herbicides, aflatoxin, and pesticides (Seebunrueng et al. 2014; Gure et al. 2015; Mitra et al. 2015), manly in environmental waters or fruits. However, VDLLME procedure has not been considered to date for the analysis of PAHs.

In our previous study, we reformed an instrument for fast pretreatment of PAHs and rapid sample collection (Wang et al. 2013). However, this instrument proved to be too expensive for routine use in many laboratories. In order to solve this problem, we created a more accessible sample collection method. Here we describe a pretreatment method of ACC combining with VDLLME that does not need special equipment. 


\section{Material and method}

\section{Instrument and solvent}

Samples analyzed were collected and extracted from a RT-flex60c marine diesel engine (Qingdao Wartsila Corporation) according to MARPOL 73/78 annex VI. PAHs emitted from marine diesel engine were determined by GC/MS (GC/MS-QP 2010 Plus, Shimadzu, Japan) equipped with a DB-5 MS capillary column $(30 \mathrm{~m} \times 0.25 \mathrm{~mm} \times 0.25 \mu \mathrm{m})$. The injection mode was splitless, and injection volume was $1 \mu \mathrm{L}$. The column flow of carrier gas (helium) was $26.2 \mathrm{~cm} / \mathrm{sec}$. The column temperature program was set at $90^{\circ} \mathrm{C}$ for $1 \mathrm{~min}$, and then raised by $25^{\circ} \mathrm{C} / \mathrm{min}$ to $180^{\circ} \mathrm{C}$, held for 0 min, raised by $5^{\circ} \mathrm{C}$ per min to $300^{\circ} \mathrm{C}$ and held for 0 min. Finally, the temperature was increased rapidly by $20^{\circ} \mathrm{C}$ per min to $320^{\circ} \mathrm{C}$ and kept for $5 \mathrm{~min}$.

Pyridine, tetrahydrofuran, hexadecyl trimethyl ammonium bromide (CTAB), tetrachloroethylene, 1,1,2,2-tetrachloroethane, and carbon tetrachloride were purchased from Merck, Germany. Methanol, ethanol, acetonitrile (HAc), acetone, acetic acid, , and sodium chloride $(\mathrm{NaCl})$ was purchursed from Kemiou Chemical, Tianjin, China. 1-hexyl-3-methylimidazolium hexafluorophosphate (HMIMPF6) were purchased from Sigma Aldrich, USA. All solvents were of analytical grade. Pure water (Millipore, Bedford, USA) was used for all the solutions and dilutions. Quantitative analysis of PAHs (2000 $\mu \mathrm{g} / \mathrm{mL}$, dichloromethane) was calibrated with a commercial standard mixture (Supelco EPA 610 PAH Mix, USA).

\section{Experimental Procedure}

1) Aqueous-capture for collection

A stainless steel joint connecting tube $(\Phi 5 \mathrm{~mm})$ was welded on the tail end of an exhaust pipe and connected with three $250 \mathrm{~mL}$ aqueous glass container to collect exhaust emissions without dilute with air. A fiber filter was set before the inlet of the brass pipe to protect the particular phase PAHs from the aqueous solution. The glass container included the optimized co-solvent CTAB $0.0002 \mathrm{~mol} / \mathrm{L}, \mathrm{NaCl} 0.01 \mathrm{~mol} / \mathrm{L}$ and $5 \%$ ethanol: $n$-hexane.

2) DLLME for concentration

A volume of $5 \mathrm{~mL}$ of sample was placed in a $15 \mathrm{~mL}$ centrifuge tube with conical bottom and was then spiked with $10 \mu \mathrm{L}$ PAHs standard solutions. After stirred $1 \mathrm{~min}, 10 \mu \mathrm{L}$ tetrachloroethylene (extraction solvent) and $1000 \mu \mathrm{L}$ acetone (disperser solvent) and was rapidly injected into the sample solution, using $1.0 \mathrm{~mL}$ syringe with a blunt needle tip, in order to induce the formation of the cloudy solution consisting of fine droplets of liquid dispersed in the sample solution. Subsequently, the mixture was vortexed for $30 \mathrm{~s}$ at the highest speed and then centrifuged for 2 min at $9000 \mathrm{rpm}$ to enhance the sedimentation of the liquid droplets. The upper phase was discarded using a $5 \mathrm{~mL}$ syringe. The lower layer was collected after dissolving in $500 \mu \mathrm{L}$ of acetone, followed by vortexing for about $30 \mathrm{~s}$. The solution was then transferred to a $1.5 \mathrm{~mL}$ vial to inject $1 \mu \mathrm{L}$ into the GC/MS equipment.

\section{Result and discussion}

\section{Bench test and sample collection}

Marine diesel engine 7RT-flex60C was used for the sampling test (specifications were listed in Table 1). Details of optimization of the ACC method for collection of gaseous PAHs have published in a previous report (Wang et al. 2013).

\begin{tabular}{ll}
\multicolumn{1}{c}{ Table $1 \quad$ Engine specifcations of 7RT-FLEX60C } \\
Model of engine & QMD-Wartsila 7RT-flex60C \\
Number of cylinder & 7 \\
Bore $\times$ stroke & $600 \mathrm{~mm} \times 2250 \mathrm{~mm}$ \\
Nominal Compression ratio & $18.4: 1$ \\
Injection pump & Electronic controlled type \\
Injector number & 3 \\
Rated Power $(\mathrm{kW})$ and Speed & $16520 \mathrm{~kW} \times 114 \mathrm{rpm}$ \\
$(\mathrm{RPM})$ & \\
\hline
\end{tabular}




\begin{tabular}{ll}
\hline Maximum Fuel Rail Pressure & 900 bar \\
Turbocharger type & ABB TPL73B12 $\times 2$ sets \\
Air cooler & SAC53F $\times 2$ sets
\end{tabular}

\section{Optimization of the DLLME method}

When selecting a suitable extraction solvent, we considered various parameters such as high density, low polarity, and the high partitioning coefficient of the PAHs in the solvent. We optimized each of these factors in pursuit of a good enrichment factor (EF) and high extraction recovery (ER). $\mathrm{ER}$ and EF were selected for evaluation of the extraction ability.
$\mathrm{EF}=\mathrm{C}_{\text {sed }} / \mathrm{C}_{\mathrm{o}}$
$\mathrm{ER}=\mathrm{C}_{\text {sed }} \times \mathrm{V}_{\text {sed }} / \mathrm{C}_{\mathrm{o}} / \mathrm{V}_{\text {aq }} \times 100 \%$

Where $\mathrm{C}_{\text {sed }}$ is the concentration of analyte in seditment; $\mathrm{C}_{\mathrm{o}}$ is the initial concentration of analyte in sample; $\mathrm{V}_{\text {sed }}$ is the sediment volume, and $\mathrm{V}_{\mathrm{aq}}$ is the sample volume.

1)Influence of the extraction solvent

Based on the above considerations, we designed the extraction solvents to contain $100 \mu \mathrm{L}$ each of the organic solvents tetrachloroethylene, HMIMPF6, 1,1,2,2-tetrachloroethane, chloroform and carbon tetrachloride. The results showed that using tetrachloroethylene resulted in the best PAHs recovery, possibly due to its high density and low water solubility. We applied tetrachloroethylene as the extracting solvent was applied at concentrations of $2 \mu \mathrm{L}, 5 \mu \mathrm{L}, 10 \mu \mathrm{L}, 15 \mu \mathrm{L}, 20 \mu \mathrm{L}$ and 25 $\mu \mathrm{L}$ to optimize extraction efficiency. We then reduced the polarity of the sample solution with $50 \%$ ethanol, thereby increasing the solubility of tetrachloroethylene. We found a low sediment phase volume when using $500 \mu \mathrm{L}$ tetrachloroethylene. EF increased noticeably with increased tetrachloroethylene volume from $5 \mu \mathrm{L}$ to $15 \mu \mathrm{L}$, where it reached the maximum value (Figure 1). Since using a solvent volume higher that $10 \mu \mathrm{L} \mathrm{mL}$ considerably decreased enrichment and the preconcentration factor, we considered $10 \mu \mathrm{L}$ tetrachloroethylene as a volume sufficient for extraction of PAHs.

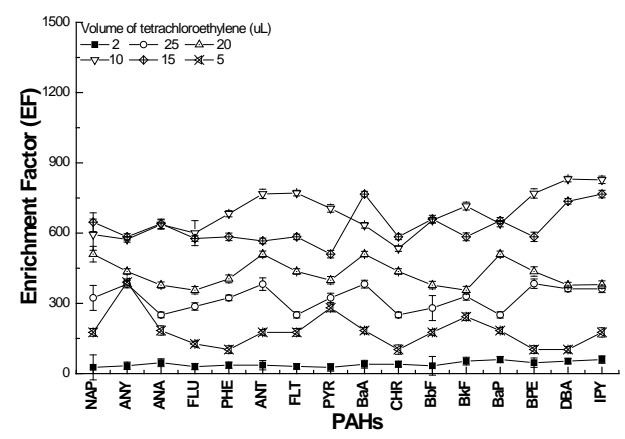

Figure 1. Effect of the volume of extracting solvent tetrachloroethylene on enrichment factor.

2) Selection of the disperser solvent

Addition of the disperser solvent produced the emulsion necessary in DLLME for developing the cloudy phase and for the transfer of PAHs from the sample solution to the sediment phase. Six volumes of acetone, $200 \mu \mathrm{L}, 500 \mu \mathrm{L}, 700 \mu \mathrm{L}, 1000 \mu \mathrm{L}, 1500 \mu \mathrm{L}$ and $2000 \mu \mathrm{L}$ were applied to optimize extraction conditions. At $1000 \mu \mathrm{L}$ acetone, the cloudy phase did not form well so as to resulting in low EF. RF reached was highest at $1000 \mu \mathrm{L}$ of acetone, and clearly decreased at 1500 $\mu \mathrm{L}$. Therefore, we selected $1000 \mu \mathrm{L}$ as the optimal volume of disperser solvent.

3) Effect of the sample $\mathrm{pH}$

In VDLLME, $\mathrm{pH}$ of the sample solution is very important. Adjust $\mathrm{pH}$ could deionized PAHs so as to reduce the solubility of PAHs. $\mathrm{PH}$ values were designed in the range from 1.0 to $4.0 \mathrm{using} \mathrm{HCl}$ or $\mathrm{NaOH}$. The highest peak areas of PAHs were obtained at pH2 (Fig. 2). At higher pH the PAHs might not be completely transformed to their neutral form, so $\mathrm{pH} 2$ was chosen as the optimum. 


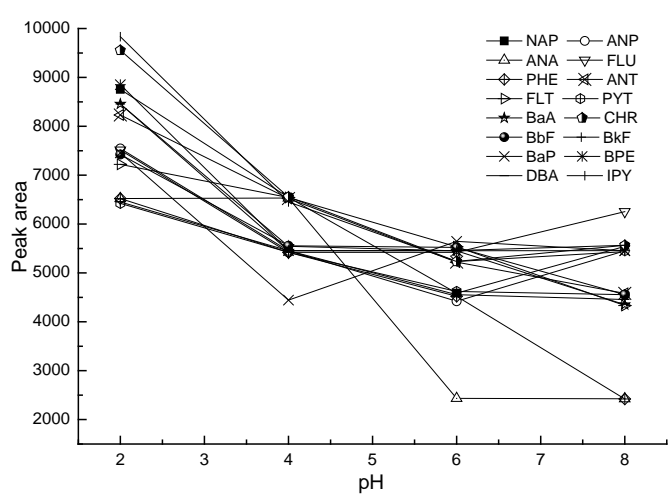

Figure 2. Effect of the sample $\mathrm{pH}$.

4) Effect of the salt addition

The addition of salts generally can reduce the solubility of the PAHs in the aqueous sample and thus enhance their partitioning into the organic phase for liquid phase. The effect of salt addition was investigated by adding $\mathrm{NaCl}$ from $0 \%$ to $20 \%(\mathrm{~m} / \mathrm{v})$. As seen in Fig. 3, peak areas of the PAHs increased slightly with the amount of salt up to $5 \%$ and then decreased, which resulted in a decrease in the peak areas. Thus, $5 \%(\mathrm{w} / \mathrm{v}) \mathrm{NaCl}$ was selected.

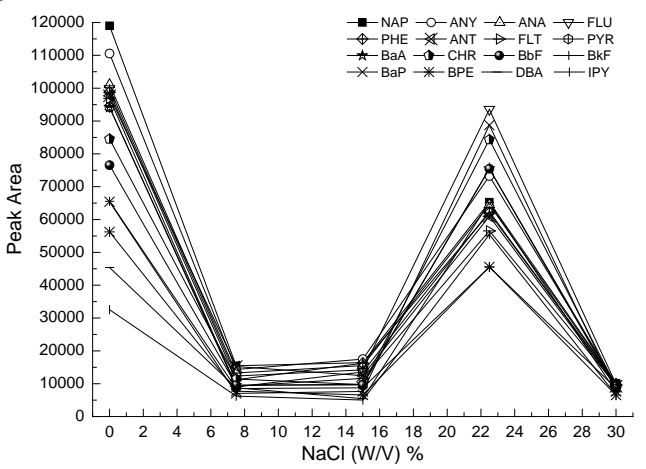

Figure 3. Effect of the salt addition.

5) Effect of vortex and extraction times

Generally, the dispersion of the extraction solvent into the sample depends on the rotational speed and vortex time. So, the vortex agitation time was varied in the range of 0-120 s. The peak areas of PAHs increased slightly till $30 \mathrm{~s}$. $30 \mathrm{~s}$ were selected in the experiments.

Extraction time was the time between the injection of disperser (containing extraction solvents) and centrifugation for DLLME. Extraction times were designed from 1-30 minutes at the speed of $9000 \mathrm{rpm}$. It was observed that the peak areas of the PAHs slightly increased up to $1 \mathrm{~min}$ and then remained constant. It was suggested that the large surface area between the aqueous and organic phases increase extraction efficiency.

\section{Methodology validation}

Our results in establishing our method are summarized in Table 2. Using this method, we found good linear relationships between peak area and concentration of PAHs in the range of 5-20 ng/L $(\mathrm{R} 2>0.9990)$. The limits of detection (LOD) of our proposed method for the targeted PAHs were in the range of $0.1-0.5 \mathrm{ng} / \mathrm{L}(\mathrm{S} / \mathrm{N}=3)$. The limit of quantitative (LOQ) was in the range of $0.2-1.0$ ng/L. The blank recovery yields ranged from $74.7 \%$ to $94.3 \%$. The result showed that GC/MS in SIM mode after ACC-DLLME was an efficient and accurate method for monitoring PAHs at very low concentration in exhaust samples.

Table 2 Method validation of capture and analysis

\begin{tabular}{llllll}
\hline PAHs & $\mathrm{R}^{2}$ & $\begin{array}{l}\text { LOD } \\
(\mathrm{ng} / \mathrm{L})\end{array}$ & $\begin{array}{l}\text { LOQ } \\
(\mathrm{ng} / \mathrm{L})\end{array}$ & $\mathrm{SR}$ & $\begin{array}{l}\mathrm{RSD} \\
(\%)\end{array}$ \\
\hline NAP & 0.9991 & 0.2 & 0.2 & 72.0 & 5.34 \\
ANY & 0.9995 & 0.5 & 0.2 & 76.3 & 2.13 \\
ANA & 0.9990 & 0.1 & 0.3 & 84.3 & 1.19 \\
FLU & 0.9990 & 0.1 & 0.5 & 79.3 & 6.75 \\
PHE & 0.9994 & 0.2 & 0.5 & 81.2 & 1.24
\end{tabular}




\begin{tabular}{llllll} 
ANT & 0.9992 & 0.2 & 0.5 & 80.5 & 2.81 \\
FLT & 0.9998 & 0.2 & 0.50 & 95.1 & 6.53 \\
PYR & 0.9997 & 0.1 & 0.50 & 88.2 & 1.43 \\
BaA & 0.9994 & 0.1 & 0.5 & 80.6 & 8.64 \\
CHR & 0.9991 & 0.1 & 0.5 & 84.4 & 1.56 \\
BbF & 0.9995 & 0.1 & 0.4 & 82.6 & 9.27 \\
BkF & 0.9994 & 0.2 & 0.2 & 80.3 & 6.68 \\
BaP & 0.9998 & 0.2 & 0.50 & 78.5 & 7.94 \\
BPE & 0.9997 & 0.5 & 1.00 & 83.3 & 3.52 \\
DBA & 0.9995 & 0.5 & 1.00 & 76.7 & 9.98 \\
IPY & 0.9995 & 0.5 & 1.00 & 72.1 & 9.36 \\
\hline
\end{tabular}

PAHs and abrreviations: Naphthalene (NAP); Acenaphthylene (ANY); Acenaphthene (ANA); Fluorene (FLU); Phenanthrene (PHE); Anthracene (ANT); Fluoranthene (FLT); Pyrene (PYR); Benz(a)anthracene (BaA); Chrysene (CHR); Benzo(b)fluoranthene (BbF); Benzo(k)fluoranthene (BkF); Benzo(a)pyrene (BaP); Benzo(g,h,i)perylene (BPE); Dibenz(a,h)anthracene (DBA): Indeno(1,2,3-cd)pyrene (IPY)

\section{Evaluation in real applications}

Spiked samples were tested to further validate the applicability of the ACC-DLLME-GC/MS method. The spiked recoveries were in the range of 72.0-95.1\% and the relative standard deviation (RSD) was 1.19-9.98\% (Table 1). All target PAHs dissolved in the aqueous solution, regardless of size, resulting in a total PAH volume of $60.75 \mu \mathrm{g} / \mathrm{m}^{3}$. To evaluate the variability of the method, the experiments were carried out for three days and subjected to three parallel tests. The average values and standard deviations were then calculated. PAHs were quantitatively captured in aqueous solution, but had great deviations in capture volumes of the semi-volatile PAHs, NAP, ANY, ANA and FLU.

\section{Comparison with traditional process}

In order to compare the result of our proposed method with traditional methods, we used a traditional method for collection and extraction of PAHs. It used a PUF method for collection (8 hours), and Soxhlet method for extraction (8 hours), KD evaporator, and GC/MS for detection resulting in a total volume of extracted PAHs of $49.59 \mu \mathrm{g} / \mathrm{m}^{3}$. This result suggests a loss of $18.37 \%$ PAHs through traditional process of collection compared to our proposed method $\left(60.75 \mu \mathrm{g} / \mathrm{m}^{3}\right)$. The proposed method was also compared with some reported method (Table 2). Since our protocol calls for a collection and pretreatment time less than 0.5 hours, our method could be completed in $1 / 10$ of the time required for traditional methods.

\section{Conclusion}

In order to evaluate gaseous phase PAHs emitted from the diesel engine, we improved a pretreatment process that coupled ACC with VDLLME. Our proposed method does not require special equipment, and could integrate the extraction, enrichment, and concentration processes into one step. Quantitative analysis of PAHs was considerably shortened compared to traditional methods and required less than 30 minutes from the sample collection to detection. Furthermore, the detection limits reached to $n g$ grade, which illustrated the high precision our method. Finally, our proposed collection and extraction method can be accomplished in $10 \%$ of the time required when using traditional methods and retain more of the volatile compounds. Therefore, we present our protocol as a more efficient method of accurate collection and extraction of PAHs from exhaust.

\section{Acknowledgements}

This research was supported by the funds of the Project of Shandong Province Higher Educational Science and Technology Program (J14LD04), Youth Foundation of Yantai University (HX12Z05). 


\section{References}

[1] Fan, C. Li, N. Cao, X.L. 2015. Determination of chlorophenols in honey samples using in-situ ionic liquid-dispersive liquid-liquid microextraction as a pretreatment method followed by highperformance liquid chromatography. Food Chemistry. 174: 446-451.

[2] Gure, A. Lara, F.J. Ana, M.G.C. et al. 2015. Vortex-assisted ionic liquid dispersive liquid-liquid microextraction for the determination of sulfonylurea herbicides in wine samples by capillary highperformance liquid chromatography. Food Chemistry. 170: 348-353.

[3] Khamphe, P. Tekasakul, S. Tekasakul, P. et al. 2013. Emissions of particulate matter and associated polycyclic aromatic hydrocarbons from agricultural diesel engine fueled with degummed, deacidified mixed crude palm oil blends. Journal of Environmental Sciences. 25(4):751-757.

[4] Mitra, A.D. Zohreh, T. Mehdi, A. et al. 2015. Application of dispersive liquid-liquid microextraction coupled with vortex-assisted hydrophobic magnetic nanoparticles based solidphase extraction for determination of aflatoxin M1 in milk samples by sensitive micelle enhanced spectrofluorimetry. Talanta. 134:98-104.

[5] Natalia, C. Pilar, V. Gema, F.M. et al. 2015. Dispersive liquid-liquid microextraction for the determination of flavonoid aglycone compounds in honey using liquid chromatography with diode array detection and time-of-flight mass spectrometry. Talanta. 131:85-191.

[6] Rezaee, M. Assadi, Y. Hosseini, M.R.M. 2006. Determination of organic compounds in water using dispersive liquid-liquid microextraction. Journal of Chromatography A. 1116(1-2):1-9.

[7] Seebunrueng, K. Santaladchaiyakit, Y. Srijaranai S. 2014. Vortex-assisted low density solvent based demulsified dispersive liquid-liquid microextraction and high-performance liquid chromatography for the determination of organophosphorus pesticides in water samples. Chemosphere. 103:51-58.

[8] Wang, M.Y. Zhang, S.J. Zhang, X. et al. 2013. Determination of Gaseous Phase Polycyclic Aromatic Hydrocarbons Emitted from a Diesel Engine by GC-MS with Novel Sample Preparation. Analytical Letters. 46(12): 1951-1961. 